\title{
On the origin of strong photon antibunching in weakly nonlinear photonic molecules
}

\author{
Motoaki Bamba, , * Atac Imamoğlu, ${ }^{2}$ Iacopo Carusotto, ${ }^{3}$ and Cristiano Ciuti, \\ ${ }^{1}$ Laboratoire Matériaux et Phénomènes Quantiques, Université Paris Diderot-Paris 7 et CNRS, \\ Bâtiment Condorcet, 10 rue Alice Domon et Léonie Duquet, 75205 Paris Cedex 13, France \\ ${ }^{2}$ Institute of Quantum Electronics, ETH Zürich, 8093 Zürich, Switzerland \\ ${ }^{3}$ INO-CNR BEC Center and Dipartimento di Fisica, Università di Trento, I-38123 Povo, Italy
}

(Dated: November 1, 2018)

\begin{abstract}
In a recent work [T. C. H. Liew and V. Savona, Phys. Rev. Lett. 104, 183601 (2010)] it was numerically shown that in a photonic 'molecule' consisting of two coupled cavities, near-resonant coherent excitation could give rise to strong photon antibunching with a surprisingly weak nonlinearity. Here, we show that a subtle quantum interference effect is responsible for the predicted efficient photon blockade effect. We analytically determine the optimal on-site nonlinearity and frequency detuning between the pump field and the cavity mode. We also highlight the limitations of the proposal and its potential applications in demonstration of strongly correlated photonic systems in arrays of weakly nonlinear cavities.
\end{abstract}

PACS numbers: 42.50.Dv, 03.65.Ud, 42.25.Hz

The photon blockade is a quantum optical effect preventing the resonant injection of more than one photon into a nonlinear cavity mode [1], leading to antibunched (sub-Poissonian) single-photon statistics. Signatures of photon blockade have been observed by resonant laser excitation of an optical cavity containing either a single atom [2] or a single quantum dot [3] in the strong coupling regime. Arguably, the most convincing realization was based on a single atom coupled to a micro-toroidal cavity in the Purcell regime [4], suggesting that the strong coupling regime of cavity-QED need not be a requirement. Concurrently, on the theory side there has been a number of proposals investigating strongly correlated photons in coupled cavity arrays 5-7] or one-dimensional optical waveguides [8]. The specific proposals based on the photon blockade effect include the fermionization of photons in one-dimensional cavity of arrays [9], the crystallization of polaritons in coupled array of cavities [10], and the quantum-optical Josephson interferometer in a coupled photonic mode system [1].

It is commonly believed that photon blockade necessarily requires a strong on-site nonlinearity $U$ for a photonic mode, whose magnitude should well exceed the mode broadening $\gamma$. However, in a recent work [12] Liew and Savona numerically showed that a strong antibunching can be obtained with a surprisingly weak nonlinearity $(U \ll \gamma)$ in a system consisting of two coupled zerodimensional (0D) photonic cavities (boxes), as shown in Fig. 1(a) [12]. Such a configuration can be obtained, e.g., by considering two modes in two photonic boxes coupled with a finite mode overlap due to leaky mirrors: the corresponding tunnel strength will be designated with $J$. In Ref. [12] numerical evidence indicated that a nearly perfect antibunching can be achieved for an optimal value of the on-site repulsion energy $U$ and for an optimal value of the detuning between the pump and mode frequency. However, a physical understanding of the mechanism leading to strong photon antibunching is needed to identify the limitations of the scheme in the context of proposed experiments on strongly correlated photons, as well as to determine the dependence of the optimal coupling and detuning on the relevant physical parameters $J$ and $\gamma$.

In this letter, we show analytically that the surprising antibunching effect is the result of a subtle destructive quantum interference effect which ensures that the probability amplitude to have two photons in the driven cavity is zero. We show that the weak nonlinearity is required only for the auxiliary cavity that is not laser driven and whose output is not monitored, indicating that photon antibunching is obtained for a driven linear cavity that tunnel couples to a weakly nonlinear one. We determine the analytical expressions for the optimal coupling $U$ and for the pump frequency detuning required to have a perfect antibunching as a function of the mode coupling $J$ and broadening $\gamma$. Our analytical results are in excellent agreement with fully numerical solutions of the master equation for the considered system. Before concluding, we discuss the experimental realization of such a scheme by using cavities embedding weakly coupled quantum dots. Moreover, we consider also the case of a ring of coupled photonic molecules showing that strong antibunching persists in presence of intersite photonic correlations.

We consider two photonic modes coupled with strength $J$; each mode has energy $E_{j}$ and an on-site photonphoton interaction strength $U_{i}(i=1,2)$. The Hamiltonian is written as

$$
\begin{aligned}
\hat{H}= & \sum_{i=1}^{2}\left[E_{i} \hat{a}_{i}^{\dagger} \hat{a}_{i}+U_{i} \hat{a}_{i}^{\dagger} \hat{a}_{i}^{\dagger} \hat{a}_{i} \hat{a}_{i}\right]+J\left(\hat{a}_{1}^{\dagger} \hat{a}_{2}+\hat{a}_{2}^{\dagger} \hat{a}_{1}\right) \\
& +F \mathrm{e}^{-\mathrm{i} \omega_{\mathrm{p}} t} \hat{a}_{1}^{\dagger}+F^{*} \mathrm{e}^{\mathrm{i} \omega_{\mathrm{p}} t} \hat{a}_{1}
\end{aligned}
$$

where $\hat{a}_{i}$ is the annihilation operator of a photon in $i$ th mode, $F$ and $\omega_{\mathrm{p}}$ are the pumping strength and fre- 

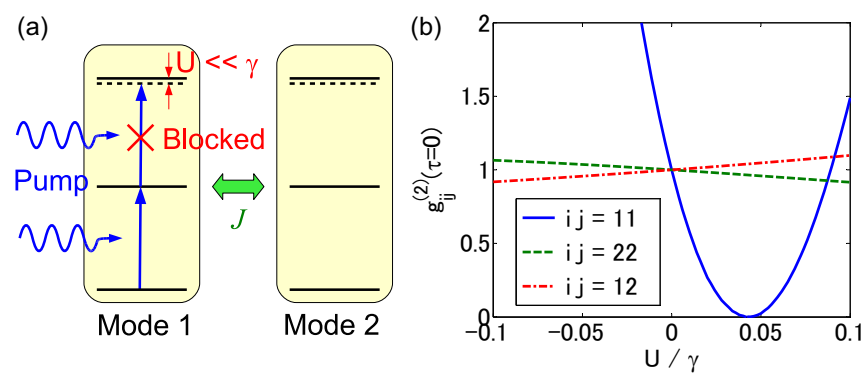

FIG. 1: (1) Sketch of the two coupled photonic modes. The coupling strength is $J$, and the antibunching is obtained with a small nonlinear energy $U$ compared to mode broadening $\gamma$. (b) Equal-time second-order correlation functions $g_{i j}^{(2)}(\tau=0)$ are plotted as functions of nonlinearity $U=U_{1}=U_{2}$ normalized to $\gamma$. The nearly perfect antibunching is obtained at the pumped mode $\left[g_{11}^{(2)}(\tau=0) \simeq 0\right]$ for $U=0.0428 \gamma$. Parameters: $\gamma_{1}=\gamma_{2}=\gamma, J=3 \gamma, E_{1}=E_{2}=\hbar \omega_{\mathrm{p}}+0.275 \gamma$, and $F_{1}=0.01 \gamma$.

quency, respectively. Following Ref. [12], we first calculate the second-order correlation function $g_{i j}^{(2)}(\tau)=$ $\left\langle\hat{a}_{i}^{\dagger} \hat{a}_{j}^{\dagger}(\tau) \hat{a}_{j}(\tau) \hat{a}_{i}\right\rangle /\left\langle\hat{a}_{i}^{\dagger} \hat{a}_{i}\right\rangle\left\langle\hat{a}_{j}^{\dagger} \hat{a}_{j}\right\rangle$ in the steady state using the master equation in a basis of Fock states [13]. The results are shown as functions of nonlinearity $U$ in Fig. 1(b). As already demonstrated in Ref. [12], we can get a strong antibunching of the pumped mode $\left(g_{11}^{(2)}(0) \simeq\right.$ 0 ) for an unexpectedly small nonlinearity $U=0.0428 \gamma$.

In order to understand the origin of the strong antibunching, we use the Ansatz

$$
\begin{aligned}
|\psi\rangle= & C_{00}|00\rangle+\mathrm{e}^{-\mathrm{i} \omega_{\mathrm{p}} t}\left(C_{10}|10\rangle+C_{01}|01\rangle\right) \\
& +\mathrm{e}^{-\mathrm{i} 2 \omega_{\mathrm{p}} t}\left(C_{20}|20\rangle+C_{11}|11\rangle+C_{02}|02\rangle\right)+\ldots,
\end{aligned}
$$

to calculate the steady-state of the coupled cavity system. Here, $|m n\rangle$ represents the Fock state with $m$ particles in mode 1 and $n$ particles in mode 2 . Under weak pumping conditions $\left(C_{00} \gg C_{10}, C_{01} \gg C_{20}, C_{11}, C_{02}\right)$, we can calculate the coefficients $C_{m n}$ iteratively. For one-particle states, the steady-state coefficients are determined by

$$
\begin{aligned}
& \left(\Delta E_{1}-\mathrm{i} \gamma_{1} / 2\right) C_{10}+J C_{01}+F C_{00}=0, \\
& \left(\Delta E_{2}-\mathrm{i} \gamma_{2} / 2\right) C_{01}+J C_{10}=0,
\end{aligned}
$$

where $\Delta E_{j}=E_{j}-\hbar \omega_{\mathrm{p}}$ and we consider a damping with rate $\gamma_{j}$ in each mode. Since we assume weak pumping, the contribution from the higher states $\left(C_{20}, C_{11}\right.$, and $\left.C_{02}\right)$ to the steady-state values of $C_{10}, C_{01}$ is negligible. From Eq. (3b), the amplitude of mode 2 can be written as

$$
C_{01}=-\frac{J}{\Delta E_{2}-\mathrm{i} \gamma_{2} / 2} C_{10}
$$

indicating that for strong photon tunneling $(J \gg$ $\left.\left|\Delta E_{2}\right|, \gamma_{2}\right)$, the probability of finding a photon in the auxiliary cavity is much larger than the driven cavity. (a)

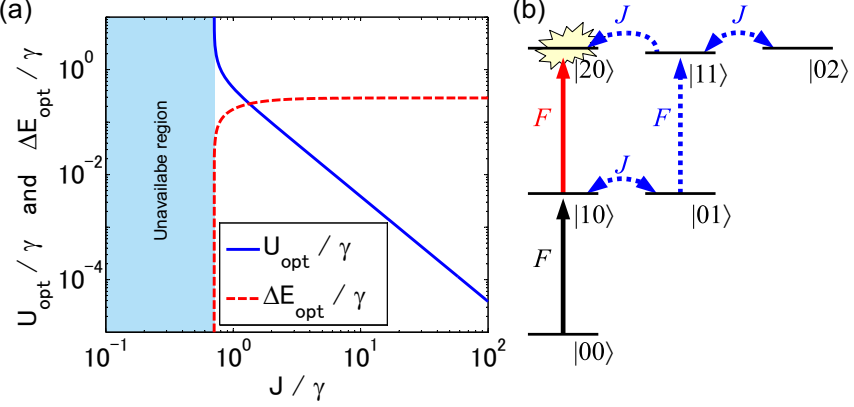

FIG. 2: (a) Optimal nonlinearity $U_{\text {opt }}$ and detuning $\Delta E_{\text {opt }}$ are plotted as functions of coupling strength $J$ normalized to $\gamma\left(\gamma_{1}=\gamma_{2}=\gamma\right.$ and $\left.E_{1}=E_{2}=E\right)$. The perfect antibunching is obtained for $J>\gamma / \sqrt{2}$. (b) Transition paths leading to the quantum interference responsible for the strong antibunching. One path is the direct excitation from $|10\rangle$ to $|20\rangle$, but it is forbidden by the interference with the other path drawn by dotted arrows.

In the same manner, the coefficients of two-particle states are determined by

$$
\begin{aligned}
& 2\left(\Delta E_{1}+U_{1}-\mathrm{i} \gamma_{1} / 2\right) C_{20}+\sqrt{2} J C_{11}+\sqrt{2} F C_{10}=0, \\
& \begin{array}{l}
\left(\Delta E_{1}+\Delta E_{2}-\mathrm{i} \gamma_{1} / 2-\mathrm{i} \gamma_{2} / 2\right) C_{11}+\sqrt{2} J C_{20}+\sqrt{2} J C_{02} \\
\quad+F C_{01}=0, \\
2\left(\Delta E_{2}+U_{2}-\mathrm{i} \gamma_{2} / 2\right) C_{02}+\sqrt{2} J C_{11}=0 .
\end{array}
\end{aligned}
$$

When we simply consider $E_{1}=E_{2}=E$, and $\gamma_{1}=\gamma_{2}=$ $\gamma$, the conditions to satisfy $C_{20}=0$ are derived from Eqs. (4) and (5) as

$$
\begin{aligned}
& \gamma^{2}\left(3 \Delta E+U_{2}\right)-4 \Delta E^{2}\left(\Delta E+U_{2}\right)=2 J^{2} U_{2}, \\
& 12 \Delta E^{2}+8 \Delta E U_{2}-\gamma^{2}=0 .
\end{aligned}
$$

For fixed $J$ and $\gamma$, from these equations, the optimal conditions (those that lead to $C_{20}=0$ ) are given by

$$
\begin{aligned}
\Delta E_{\mathrm{opt}} & = \pm \frac{1}{2} \sqrt{\sqrt{9 J^{4}+8 \gamma^{2} J^{2}}-\gamma^{2}-3 J^{2}}, \\
U_{\mathrm{opt}} & =\frac{\Delta E_{\mathrm{opt}}\left(5 \gamma^{2}+4 \Delta E_{\mathrm{opt}}{ }^{2}\right)}{2\left(2 J^{2}-\gamma^{2}\right)}
\end{aligned}
$$

and, if $J \gg \gamma$, they are approximately written as

$$
\begin{aligned}
\Delta E_{\mathrm{opt}} & \simeq \frac{\gamma}{2 \sqrt{3}}, \\
U_{\mathrm{opt}} & \simeq \frac{2}{3 \sqrt{3}} \frac{\gamma^{3}}{J^{2}} .
\end{aligned}
$$

In Fig. 2(a), the optimal $\Delta E_{\text {opt }}$ and $U_{\text {opt }}$ [Eq. (7)] are plotted as functions of $J / \gamma$. The strong antibunching can be obtained even if $U_{2}<\gamma$, provided $J>\gamma / \sqrt{2}$. Remarkably, the required nonlinearity decreases with increasing tunnel coupling $J$ obeying Eq. 8b . 


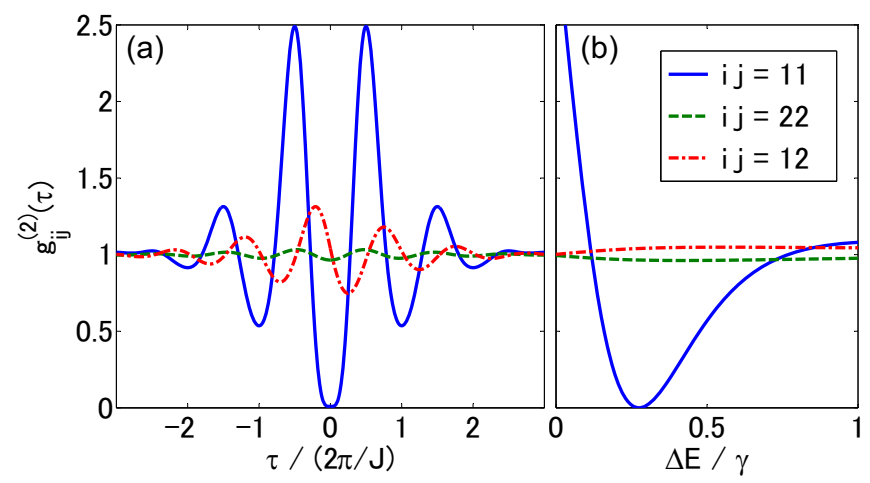

FIG. 3: (a) The time evolution of the second-order correlation function, which oscillates with period $2 \pi / J$ as the result of amplitude oscillation between $|01\rangle$ and $|10\rangle$. (b) Equal-time second-order correlation functions are plotted as functions of $\Delta E_{1}=\Delta E_{2}=\Delta E$ normalized to $\gamma_{1}=\gamma_{2}=\gamma$. The spectral width of the antibunching resonance is $\approx 0.3 \gamma$. Parameters: $J=3 \gamma, U_{1}=U_{2}=0.0428 \gamma$, and $F=0.01 \gamma . \Delta E=0.275 \gamma$ in panel (a).

In Fig. 2(b), we show a sketch of the quantum interference effect responsible for this counter-intuitive photon antibunching. The interference is between the following two paths: (a) the direct excitation from $|10\rangle \stackrel{F}{\rightarrow}|20\rangle$ (solid arrow) and (b) tunnel-coupling-mediated transition $|10\rangle \stackrel{J}{\leftrightarrow}|01\rangle \stackrel{F}{\rightarrow}(|11\rangle \stackrel{J}{\leftrightarrow}|02\rangle) \stackrel{J}{\rightarrow}|20\rangle$ (dotted arrows). In order to show in detail the origin of the quantum interference, we rewrite Eqs. (6) for $C_{20}=0$ as follows. First, we calculate $C_{11}$ from Eqs. (41) and (5) neglecting $C_{20}$ as

$$
\begin{aligned}
C_{11}= & -2 J F C_{10}\left(\Delta E+U_{2}-\mathrm{i} \gamma / 2\right)(\Delta E-\mathrm{i} \gamma / 2)^{-1} \\
& {\left[2 J^{2}-4 \Delta E\left(\Delta E+U_{2}\right)+\gamma^{2}+\mathrm{i} 2 \gamma\left(2 \Delta E+U_{2}\right)\right]^{-1} . }
\end{aligned}
$$

This amplitude is the result of excitation from $|01\rangle$ to $|11\rangle$ and of the coupling between $|10\rangle$ and $|01\rangle$ and also between $|11\rangle$ and $|02\rangle$. From this amplitude, $C_{20}$ is determined by Eq. (5a) as $C_{20} \propto J C_{11}+F C_{10}$, and we can derive Eqs. (6) by the condition $C_{20}=0$.

As seen in Fig. 1(b), while no more than one photon is present in the first cavity mode at the optimal condition, there can be more than one photons in the whole system. While there is nearly perfect antibunching in the driven mode $\left[g_{11}^{(2)}(\tau=0)<<1\right]$, the cross-correlation between the two modes exhibits bunching $\left[g_{12}^{(2)}(\tau=0)>1\right]$. The amplitude oscillation between $|10\rangle$ and $|01\rangle$ produces the time oscillation of $g_{11}^{(2)}(\tau)$ with period $2 \pi / J$ as reported in Ref. [12] and shown in Fig. 3(a).

The equal-time correlation functions is plotted in Fig. 3(b) as a function of the pump detuning $\Delta E / \gamma$ : while the optimal value of the detuning is at $\Delta E=$ $0.275 \gamma$, a strong antibunching is obtained in a range of about $0.3 \gamma$ around the optimal value and the width of this

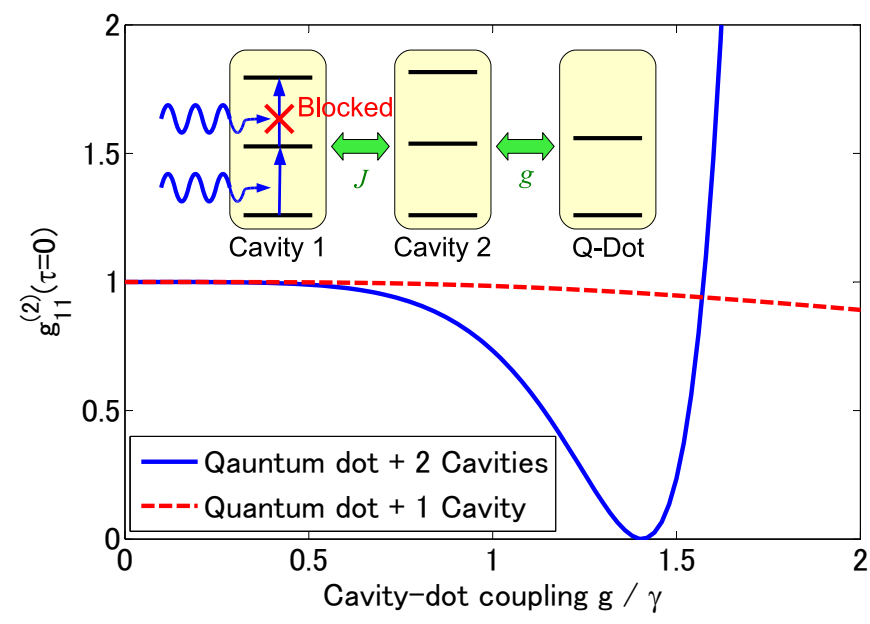

FIG. 4: Equal-time correlation functions are plotted as functions of coupling strength $g$ between a cavity and a quantum dot. Solid line represents the results in the system sketched in the inset [Eq. [10)]. Parameters: $\gamma_{1}=\gamma_{2}=\gamma_{\mathrm{ex}}=\gamma$, $J=3 \gamma, E_{1}=\hbar \omega_{\mathrm{p}}+0.275 \gamma, E_{2}-E_{1}=\gamma, E_{\text {ex }}-E_{2}=2 \gamma$ and $F=0.01 \gamma$. Dashed line represents the result in the system with one quantum dot and one cavity [Jaynes-Cummings model]. $\gamma_{1}=\gamma_{\mathrm{ex}}=\gamma, E_{\mathrm{ex}}-E_{1}=2 \gamma, F=0.01 \gamma$, and $\hbar \omega_{\mathrm{p}}$ is tuned to the lower one-particle eigenenergy of the JaynesCummings ladder.

window does not significantly depend on $J / \gamma$. This may suggest that pump pulses of duration $\Delta t_{p}$ longer than $1 /(0.3 \gamma)$ could be enough to ensure strong antibunching. However, the timescale over which strong quantum correlations between the photons exist is on the order of $1 / J<\sqrt{2} / \gamma$, as seen in Fig. B(a). While weak nonlinearities do lead to strong quantum correlations, these correlations last for a timescale that scales with $1 / J \propto \sqrt{U_{\mathrm{opt}}}$ (see Eq. (8b) ). From a practical perspective, a principal difficulty with the observation of the photon antibunching with weak nonlinearities is that it requires fast singlephoton detectors [14]. Conversely, for a given detection set-up, the required minimal value of the nonlinearity is ultimately determined by the time resolution of the available single photon detector.

As seen in Eq. (6), the nonlinearity $U_{1}$ of the pumped cavity mode is not essential for the antibunching. This means that only the auxiliary (undriven) photonic mode must have a (weak) nonlinearity to achieve the quantum interference leading to perfect photon antibunching. As a practical realization, one could consider two coupled photonic crystal nanocavities, where the auxiliary cavity contains a single quantum dot that leads to the required weak nonlinearity (see the inset in Fig. 44). The Hamil- 
(a)

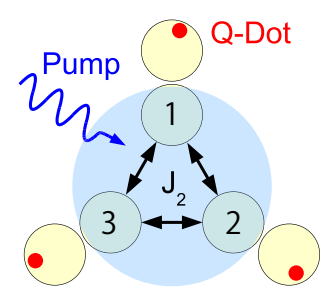

(b)

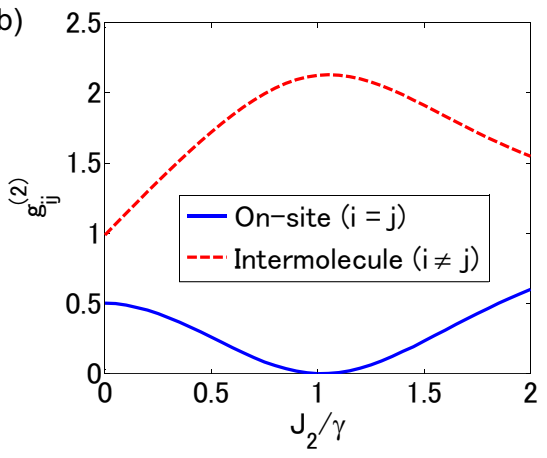

FIG. 5: (a) Sketch of a triangular lattice of coupled photonic 'molecules'. The driven cavities $(i=1,2$, and 3$)$ are coupled with strength $J_{2}$. (b) The equal-time second-order correlation functions in each mode (solid line) and between neighbors (dashed line) are plotted versus $J_{2} / \gamma$. Parameters: $J=3 \gamma$, $E_{1}=\hbar \omega_{\mathrm{p}}+0.450 \gamma, U=0.0769 \gamma$ and $F=0.01 \gamma$.

tonian is written as

$$
\begin{aligned}
\hat{H}_{\text {cav-JC }}= & \sum_{i=1}^{2} E_{i} \hat{a}_{i}^{\dagger} \hat{a}_{i}+J\left(\hat{a}_{1}^{\dagger} \hat{a}_{2}+\hat{a}_{2}^{\dagger} \hat{a}_{1}\right) \\
& +E_{\text {ex }}|\operatorname{ex}\rangle\langle\operatorname{ex}|+g\left(\hat{a}_{2}^{\dagger}|\mathrm{g}\rangle\langle\mathrm{ex}|+\text { H.c. }\right) \\
& +F \mathrm{e}^{-\mathrm{i} \omega_{\mathrm{p}} t} \hat{a}_{1}^{\dagger}+F^{*} \mathrm{e}^{\mathrm{i} \omega_{\mathrm{p}} t} \hat{a}_{1} .
\end{aligned}
$$

Here, $|\mathrm{g}\rangle$ and $|\mathrm{ex}\rangle$ represent the ground and excited states of the quantum dot, respectively, $E_{\text {ex }}$ is the excitation energy, and $g$ is the coupling energy with cavity mode 2. Since the required nonlinearity is relatively weak, one can use a quantum dot which is off-resonant with respect to the cavity mode $\left(\left|E_{\mathrm{ex}}-E_{2}\right|>\gamma_{2}=\gamma\right)$ and/or does not satisfy strong coupling condition $(g \simeq \gamma)$. We take the quantum dot exciton broadening to be equal to the cavity decay rate for simplicity. We have solved numerically the master equation associated to the Hamiltonian in Eq. (10). Fig. 目 shows $g_{11}^{(2)}(\tau=0)$ of the pumped mode as a function of $g / \gamma$. The coupling energy between the two cavities is $J=3 \gamma$, and then the required nonlinear energy should be $U_{\mathrm{opt}}=0.0428 \gamma$ from Fig. 2. In the present system, this nonlinear energy is practically achieved at $g=1.4 \gamma$, which is an intermediate strength between the weak- and strong-coupling regime of cavity mode and quantum dot excitation. The dashed line in Fig. 4 represents the results in the system consisting of one quantum dot and one cavity: in this ordinary JaynesCummings system, only a small antibunching is obtained at $g \simeq \gamma$, and the strong-coupling $g \gg \gamma$ is required for the observation of large photon antibunching [1, 2]. In contrast, in the new scheme using the quantum interference, a nearly perfect antibunching can be obtained even for $g \simeq \gamma$.

Finally, we note that the quantum interference can be generalized to a system of many coupled photonic molecules: in this case, the strong on-site antibunching

can show an interesting interplay with quantum correlation between neighboring photonic modes. As a demonstration, we consider a ring of three molecules whose driven dots are coupled with each other by a tunnel coupling of amplitude $J_{2}$ [see Fig. 5(a)]. Also in this case a nearly perfect antibunching can be observed in each driven mode, as shown in the plots of $g_{i i}^{(2)}(\tau=0)$ as a function of $J_{2} / \gamma$ that are shown as a solid line in Fig. 5(b). In order to optimize the antibunching at a finite value of $J_{2} \simeq \gamma$, values of $U=0.0769 \gamma$ and $\Delta E=0.450 \gamma$ slightly different from the single-molecule optimal ones $\left(U_{\mathrm{opt}}=0.0428 \gamma\right.$ and $\left.\Delta E_{\mathrm{opt}}=0.275 \gamma\right)$ had to be chosen. At the same time, a strong bunching effect is observed in the equal-time cross-correlation function between neighboring cavities, which shows a value of $g_{i \neq j}^{(2)}(0)$ significantly larger than the coherent field value of $g_{i \neq j}^{(2)}(0)=1$. This remarkable combination of strong on-site antibunching and strong inter-site bunching suggests that this system may be a viable alternative to the realization of a Tonks-Girardeau gas of fermionized photons discussed in Ref. [9].

In summary, we have analytically determined that a destructive quantum interference mechanism is responsible for strong antibunching in a system consisting of two coupled photonic modes with small nonlinearity $(U<\gamma)$. The quantum interference effect occurs for an optimal onsite nonlinearity $U_{\mathrm{opt}} \simeq \frac{2}{3 \sqrt{3}} \frac{\gamma^{3}}{J^{2}}$, where $J$ is the intermode tunnel coupling energy and $\gamma$ is the mode broadening. This robust quantum interference effect has the peculiar feature that the resulting quantum correlation between the generated photons survive for timescales much shorter than the photon lifetime. Nonetheless, we have shown that this quantum interference scheme has the potential to generate strongly correlated photon states in arrays of weakly nonlinear cavities.

* E-mail: motoaki.bamba@univ-paris-diderot.fr

† E-mail: cristiano.ciuti@univ-paris-diderot.fr

[1] A. Imamoğlu, H. Schmidt, G. Woods, and M. Deutsch, Phys. Rev. Lett. 79, 1467 (1997).

[2] K. M. Birnbaum, A. Boca, R. Miller, A. D. Boozer, T. E. Northup, and H. J. Kimble, Nature 436, 87 (2005).

[3] A. Faraon, I. Fushman, D. Englund, N. Stoltz, P. Petroff, and J. Vuckovic, Nat. Phys. 4, 859 (2008).

[4] B. Dayan, A. S. Parkins, T. Aoki, E. P. Ostby, K. J. Vahala, and H. J. Kimble, Science 319, 1062 (2008).

[5] M. J. Hartmann, F. G. S. L. Brandao, and M. B. Plenio, Nat. Phys. 2, 849 (2006).

[6] A. D. Greentree, C. Tahan, J. H. Cole, and L. C. L. Hollenberg, Nat. Phys. 2, 856 (2006).

[7] D. G. Angelakis, M. F. Santos, and S. Bose, Phys. Rev. A 76, 031805 (2007).

[8] D. E. Chang, V. Gritsev, G. Morigi, V. Vuletic, M. D. Lukin, and E. A. Demler, Nat. Phys. 4, 884 (2008). 
[9] I. Carusotto, D. Gerace, H. E. Tureci, S. De Liberato, C. Ciuti, and A. Imamoğlu, Phys. Rev. Lett. 103, 033601 (2009).

[10] M. J. Hartmann, Phys. Rev. Lett. 104, 113601 (2010).

[11] D. Gerace, H. E. Tureci, A. Imamoglu, V. Giovannetti, and R. Fazio, Nat. Phys. 5, 281 (2009).

[12] T. C. H. Liew and V. Savona, Phys. Rev. Lett. 104, 183601 (2010).

[13] A. Verger, C. Ciuti, and I. Carusotto, Phys. Rev. B 73,
193306 (2006).

[14] In a single nonlinear cavity, the requirement for fast photon detection can be avoided by using pulsed-laser excitation; this approach does not work in the system we analyze due to small bandwidth of the nonlinearity, i.e., the available time of the antibunching is shorter than the temporal width of pump pulse $\left(1 / J<\Delta t_{\mathrm{p}}\right)$ 